\title{
Substantia Nigra Activity Level Predicts Trial-to-Trial Adjustments in Cognitive Control
}

\author{
Carsten Nicolas Boehler ${ }^{1,2,3}$, Nico Bunzeck ${ }^{4}$, Ruth M. Krebs ${ }^{3}$, \\ Toemme Noesselt ${ }^{2,4,5}$, Mircea A. Schoenfeld ${ }^{1,2,5}$, Hans-Jochen Heinze ${ }^{1,2,5}$, \\ Thomas F. Münte ${ }^{2,5}$, Marty G. Woldorff ${ }^{3}$, and Jens-Max Hopf $\mathbf{f}^{1,2,5}$
}

\begin{abstract}
Effective adaptation to the demands of a changing environment requires flexible cognitive control. The medial and the lateral frontal cortices are involved in such control processes, putatively in close interplay with the BG. In particular, dopaminergic projections from the midbrain (i.e., from the substantia nigra $[\mathrm{SN}]$ and the ventral tegmental area) have been proposed to play a pivotal role in modulating the activity in these areas for cognitive control purposes. In that dopaminergic involvement has been strongly implicated in reinforcement learning, these ideas suggest functional links between reinforcement learning, where the outcome of actions shapes behavior over time, and cognitive control in a more general context, where no direct reward is involved. Here, we provide evidence from functional MRI in humans that activity in the SN predicts sys-
\end{abstract}

\section{INTRODUCTION}

Effective behavioral adaptation to the demands of a changing environment requires flexible cognitive control. Physiologically, the medial and the lateral frontal cortices have been frequently linked to such control processes, putatively in close interplay with the BG (Frank, Woroch, \& Curran, 2005; Pasupathy \& Miller, 2005; Holroyd \& Coles, 2002; Botvinick, Braver, Barch, Carter, \& Cohen, 2001; Gehring \& Knight, 2000; Carter et al., 1998). Preceding actual control adjustments, the need for behavioral adaptation has to be detected, and various studies have related the ACC to this process, although its actual role in this context is not settled (Botvinick, Cohen, \& Carter, 2004).

Irrespective of where the necessity to adapt control settings is initially registered, the control signals have to be directed to the relevant areas that mediate behavioral adaptations. Prominent theoretical accounts posit that a control signal from the midbrain may induce adjustments in brain areas that actually implement the cognitive con-

\footnotetext{
${ }^{1}$ Leibniz Institute for Neurobiology, Magdeburg, Germany, ${ }^{2}$ Otto-von-Guericke-University, Magdeburg, Germany, ${ }^{3}$ Duke University, Durham, NC, ${ }^{4}$ University College London, United Kingdom, ${ }^{5}$ Center for Behavioral Brain Sciences, Magdeburg, Germany
}

tematic subsequent trial-to-trial RT prolongations that are thought to reflect cognitive control in a stop-signal paradigm. In particular, variations in the activity level of the SN in one trial predicted the degree of RT prolongation on the subsequent trial, consistent with a modulating output signal from the SN being involved in enhancing cognitive control. This link between SN activity and subsequent behavioral adjustments lends support to theoretical accounts that propose dopaminergic control signals that shape behavior both in the presence and in the absence of direct reward. This SN-based modulatory mechanism is presumably mediated via a wider network that determines response speed in this task, including frontal and parietal control regions, along with the BG and the associated subthalamic nucleus.

trol. In particular, dopaminergic projections from the midbrain (i.e., mainly the substantia nigra $[\mathrm{SN}]$ and the ventral tegmental area [VTA]) to the frontal cortex and the BG have been proposed to play a pivotal role in modulating activity in these areas for cognitive control purposes (Brown \& Braver, 2005; Frank et al., 2005; Montague, Hyman, \& Cohen, 2004; Ridderinkhof, Ullsperger, Crone, \& Nieuwenhuis, 2004; Ridderinkhof, van den Wildenberg, Segalowitz, \& Carter, 2004; Holroyd \& Coles, 2002). These accounts explicitly emphasize commonalities to a dopaminergic "teaching signal" that has been suggested to underlie reinforcement learning (e.g., Schultz, 2000). Reinforcement-learning theories posit that actions leading to reward are reinforced by a phasic increase in dopaminergic neuronal activity, whereas actions that repeatedly fail to yield reward are associated with a phasic suppression of dopaminergic activity, indicating the need for behavioral adjustment. Several computational models have proposed a similar mechanism for cognitive control even in the absence of direct reward. In these models, conditions leading to stronger cognitive control, such as the commission of errors, are taken to be similar to conditions of reward omission, with both leading to subsequent behavioral adjustments (Brown \& Braver, 2005; Frank et al., 2005; Holroyd \& Coles, 2002; Braver \& Cohen, 2000). Following this notion, conditions of stronger cognitive 
control would be expected to be preceded by lower activity in the SN or VTA.

Here, we used the well-established stop-signal paradigm (Figure 1A; Logan, 1994) to investigate the relationship between specific neural activity elicited by different trial types and subsequent behavioral adaptation (Figure 1B). In this paradigm, which consists of frequent go trials (GTs) and less frequent stop trials (STs), systematic RT prolongations have frequently been reported on trials following an ST, presumably indicative of a modulation of cognitive control (Boehler et al., 2009; Enticott, Bradshaw, Bellgrove, Upton, \& Ogloff, 2009; Li et al., 2008). Addressing the neural mechanisms related to these sequential behavioral adjustments, we used fMRI (Figure 1C) to examine the relationship between the neural activity elicited by stop and GTs to the RT on the subsequent GT. Given the prediction that lower dopamine neuron activity signals the need for stronger cognitive control, we hypothesized an inverse relationship between GT RTs and SN or VTA activity elicited by preceding STs but not by preceding GTs (Figure 1D).

\section{METHODS}

\section{Participants}

Twelve subjects participated in this study ( 6 women, mean age $=24.5$ ). All subjects had correct or corrected-tonormal visual acuity, and none of them reported a history of psychiatric or neurological disorders. All gave written informed consent and were paid for participation. The experiment was approved by the ethics committee of the Otto-von-Guericke University Magdeburg.

\section{Task}

The task in this experiment was adopted from a stop-signal paradigm used in an earlier magneto-encephalographic study (Figure 1A; Boehler et al., 2009); it only differed in the between-trial timing to meet the requirements of fMRI. The stop-signal paradigm employs two types of trials that are presented in a random sequence: the frequent GTs, where a response to a choice-reaction stimulus is required, and the less-frequent STs, where the presentation of a stop-signal rapidly succeeding the choice-reaction stimulus indicates that the response needs to be stopped. In GTs, which accounted for $60 \%$ of all trials, a green German traffic light symbol was presented for $800 \mathrm{msec}$, and subjects had to decide whether it was oriented to the left or right (mapped to the right index and middle finger; the task-relevant stimulus was surrounded by four task-irrelevant green traffic light signs of random left/right orientation (not depicted in Figure 1A; see Boehler et al., 2009). STs (20\% of trials) started identically to GTs, but after a certain SOA the go symbol was replaced by a red stop sign. (Additional control conditions reported in Boehler et al., 2009, that mimicked the visual stimulation of GTs and STs were presented in 20\% of the trials; these randomly occurring trials were modeled as covariates of no interest and will not be further discussed here, as the analysis focuses exclusively on GTs that were preceded by either another GT or by an ST.) The stop sign signaled subjects to withhold their response. The SOA between the choice-reaction stimulus and the stop sign is an important factor determining whether subjects accomplish withholding the motor response (called successful STs [SST])
Figure 1. Paradigm, analysis, and data acquisition. (A) In the stop-signal paradigm, a choice-reaction stimulus (here a German traffic light sign oriented to the left or right) is either presented during the entire trial (GT) or substituted by a stop signal (ST) after a certain time delay that is set trial to trial by a tracking algorithm. (Additional irrelevant flanking items of random orientation were present as in Boehler et al., 2009; see Methods.) This stop signal indicates to withhold the triggered response, yielding SST and UST. (B) This study focuses on behavioral adaptations in GTs following USTs (UsT GT), SSTs (ssTGT), or GTs (GTGT). (C) Approximate slice orientation and extent of the acquired partial volume

overlaid on the MT template. (D) We specifically hypothesized that the activity level in the SN or VTA during STs would influence the RT in the subsequent GT, with low activity leading to slowed subsequent responses and vice versa (the ellipsoids represent activity related to three different STs).

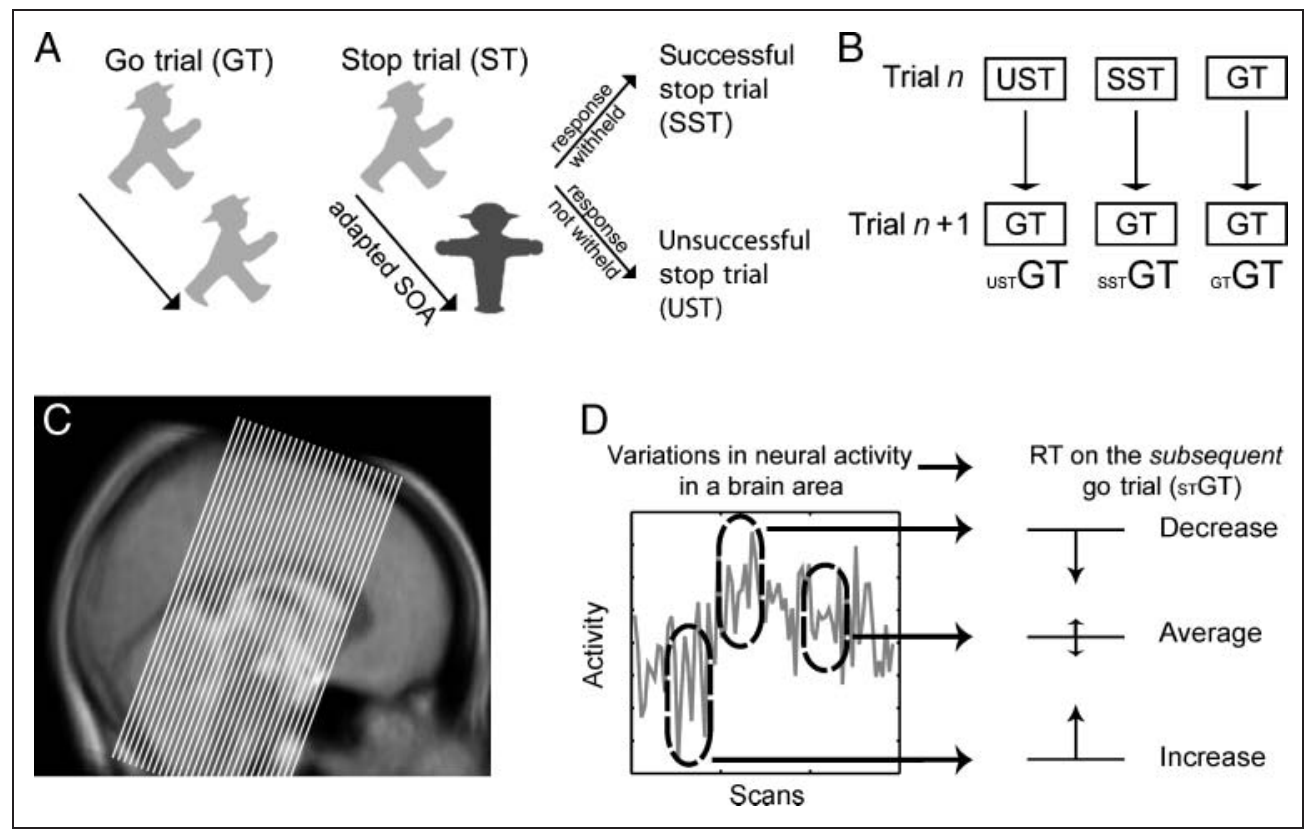


or fail to inhibit their response (called unsuccessful STs [UST]; see Logan, 1994). Therefore, the timing of the stop signal is usually titrated so as to yield an approximately equivalent number of SST and UST, by on-line adaptation of each subject's individual SOA between the choicereaction stimulus and the stop sign. Specifically, the SOA was increased by $17 \mathrm{msec}$ after an SST and decreased by the same amount after a UST. The initial SOA was $150 \mathrm{msec}$, and the total stimulus duration was kept constant at $800 \mathrm{msec}$. A total of 1735 trials was presented, divided between 10 runs. The intertrial interval varied pseudorandomly between 1.5 and 6 sec following a gamma function to allow for the separation of different conditions in an event-related fMRI analysis.

\section{Data Acquisition}

The fMRI data were acquired on a 3-T MRI system (Siemens Magnetom Trio, Erlangen, Germany) with EPI using a circularly polarized eight-channel head coil (Bruker, Ettlingen, Germany). In the functional runs, slices were acquired parallel to the brainstem in an odd-even interleaved direction that covered the midbrain, the temporal lobe, the parts of the frontal cortex, and the cerebellum (Figure 1C). Twenty-four T2*-weighted images (EPI sequence) per volume sensitive to BOLD contrast were obtained (matrix size $=64 \times 64,24$ slices per volume, field of view $=192 \times 192 \mathrm{~mm}$, spatial resolution $=3 \times 3 \times 3 \mathrm{~mm}$, gap $=0.3 \mathrm{~mm}$, echo time $[\mathrm{TE}]=30 \mathrm{msec}$, repetition time $[\mathrm{TR}]=1500 \mathrm{msec}$, flip angle $=75^{\circ}$ ). For each subject, functional data were acquired in 10 runs, each containing 252 volumes. Six additional volumes per run were acquired at the beginning of each functional run and subsequently discarded from the analysis to allow for steadystate magnetization. In addition, structural images of each subject's entire brain were collected by T1-weighted inversion recovery prepared EPI (IR-EPI) sequences (matrix size $=64 \times 64$, 60 slices, field of view $=192 \times 192 \mathrm{~mm}$, spatial resolution $=3 \times 3 \times 3 \mathrm{~mm}$, gap $=0.3 \mathrm{~mm}, \mathrm{TE}=$ $33 \mathrm{msec}$, inversion time $=1450 \mathrm{msec}, \mathrm{TR}=15000 \mathrm{msec}$ ).

\section{Data Analysis}

The fMRI data were preprocessed and statistically analyzed using the SPM5 software package (Wellcome Department of Imaging Neuroscience, Institute of Neurology, London, UK) and MATLAB 7.0 (The MathWorks, Inc., Natick, MA). All functional images were corrected for odd/even slice intensity differences with reference to the middle slice acquired in time, corrected for motion artifacts by realignment to the first volume, spatially normalized to a standard T1-weighted SPM template (Ashburner \& Friston, 1999) by warping the subjects anatomical IR-EPI to the SPM template and by applying these parameters to the functional images. The functional images were then resampled to $2 \times 2 \times 2 \mathrm{~mm}$ and smoothed with an isotropic 4-mm FWHM Gaussian kernel, and the time-series fMRI data were high-pass filtered (cutoff $=128 \mathrm{sec}$ ). For each subject, a statistical model was computed by applying a canonical hemodynamic response function (HRF) combined with time and dispersion derivatives for each of the conditions (Friston et al., 1998). To capture residual movement-related artifacts, six covariates were included (the three rigid-body translation and the three rotations resulting from realignment) as regressors of no interest.

\section{RT Regressors}

To fit hemodynamic responses with RTs on a trial-to-trial basis, parametric modulators were introduced into the analysis (Yarkoni, Barch, Gray, Conturo, \& Braver, 2009; Weissman, Roberts, Visscher, \& Woldorff, 2006; Buchel, Holmes, Rees, \& Friston, 1998). To this end, RT variations around each subject's mean RT were extracted for each trial and standardized across trials. These values were then convolved with the canonical HRF for the respective or preceding trial (see next paragraph) and entered into the model as an additional class of basis functions that are orthogonal to those representing the canonical HRF. Importantly, both types of basis functions were estimated in the same model so that the canonical HRF responses account for general differences between the conditions, whereas the RT regressors model the response variations in the different conditions as a function of RT. In essence, the RT regressors will identify areas whose activity variations correlate with the variations of the RTs from trial to trial around the mean RT for that subject. Compared with approaches that separate and contrast trials into conditions with different RTs (e.g., a median split), this approach has the advantage of taking into account the whole trial-to-trial variability of the hemodynamic and behavioral responses, thus identifying brain areas that carry the same fluctuation pattern as the behavioral variable under study (Figure 1D).

\section{Different Statistical Models}

Two statistical models were estimated for each subject, allowing assessment of different effects related to withintrial activity and across-trial adaptations. The labeling of trial-types is based on two different time-frames: (1) SST, UST, and GT reflect the conditions within a given trial,

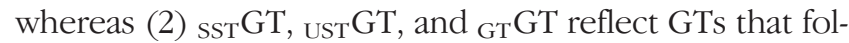
low an SST, a UST, or a GT, respectively, thus only differing in trial history. The descriptions either refer to the fMRI data (functional) or to the RT data (behavioral):

(i) The " $f / b$ " model (functional and behavioral data from the same current trial) fits the hemodynamic response of the different trial types with the corresponding RT regressor from that same trial (i.e., GT and UST, but not for SST where no RTs existed because of the successful withholding of the response). 
(ii) The " $f /(b+1)$ " model (functional data of one trial and behavioral data of the next GT) fits the hemodynamic responses of SST, UST, and GT (that are followed by a GT) with the RT fluctuations of that subsequent GT (sST GT, UST GT, and ${ }_{\mathrm{GT}} \mathrm{GT}$ ). This analysis thus captures the influence of brain activity in one trial on the RT in the next trial.

In both models, only correct GTs and STs were analyzed, whereas all other trial types were modeled as regressors of no interest. Furthermore, in the $f /(b+1)$ model, only trials were included into the relevant conditions that were succeeded by a correct GT. The parameter estimates resulting from each condition/contrast and subject (first-level analysis) were entered into a second-level random-effects group analysis using one-sample $t$ tests (thresholded at $p<.001$ and $k=4$ contiguous voxels for the midbrain and $k=10$ contiguous voxels for activations outside of the midbrain). In addition, $p$ value correction was performed using Gaussian field theory with respect to the whole acquired volume (thresholded at an uncorrected $p$ value level of $p<.001$ ), and results that were significant on the cluster level $(p<.05)$ are highlighted in the result tables. The significance of the activated clusters in the $\mathrm{SN}$ was assessed by using small volume correction (SVC; Worsley et al., 1996) with respect to a manual segmentation of the bilateral $\mathrm{SN}$. To further account for the small volume, ROI analyses in the SN were performed on the unsmoothed data of the single subjects. Functional parameter estimates were extracted using the MarsBar software package (http://marsbar.sourceforge. net/). The ROI that was used to characterize activity in the $\mathrm{SN}$ was determined from the average activity of all three parametric modulators of interest in the $f /(b+1)$

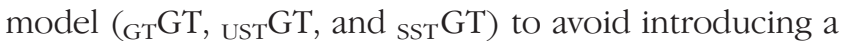
bias for any condition. This analysis was thresholded at $p<.01$, identifying an eight-voxel cluster in the right SN. Because of the complete lack of any activity in the SN in the $f / b$ model, this ROI was also used to extract activity estimates for the $f / b$ model. To verify the anatomical localization of structures within the midbrain, the activation maps were superimposed on a magnetization transfer (MT) template, which was derived from averaging the normalized MT image of 33 young adults (Bunzeck \& Duzel, 2006). On MT images, the SN region can be distinguished from surrounding structures as a bright stripe whereas the adjacent red nucleus appears dark. Activation maps were overlaid on the anatomical data using MRIcro (http://www. sph.sc.edu/comd/rorden/mricro.html). Statistical assessment of the behavioral and ROI data was accomplished by means of paired $t$ tests and one-sample $t$ tests against zero.

\section{Control Experiment}

Eighteen subjects participated in this study, of which two had to be excluded because of technical problems (of the 16 remaining participants, 9 were women; mean age $=$
22.8 years). All subjects had correct or corrected-to-normal visual acuity, and none of them reported a history of psychiatric or neurological disorders. All were paid for participation and gave written informed consent before the experiment in accordance with the Duke University institutional review board.

The experiment consisted of two types of trial blocks, each containing $50 \%$ of the trials. In one type of trial blocks, the task was identical to the main experiment, and respective data will be the focus here. The other type of trial blocks was identical regarding trial structure, but subjects were instructed to ignore stop signals and to respond on all trials. The results of these latter trial blocks will not be reported here. The former trial blocks were identical to the main experiment except for two minor modifications: (1) there were no flanking items around the target, and (2) there were no additional control conditions (yielding $80 \%$ GTs and 20\% STs). Both aspects are very unlikely to affect the results of the main experiment, but a replication of our main finding with this paradigm would additionally rule out any influence of these factors. A total of approximately 470 trials was presented in the trial blocks analyzed here.

MR data were acquired on a 3-T GE Signa MRI system. High-resolution structural T1 (3D Fast Spoiled Gradient Recalled (FSPGR); $1 \times 1 \times 1 \mathrm{~mm}$ resolution) and proton density/T2-weighted images (2-D Fast Spin Echo (FSE); $1 \times 1 \times 1 \mathrm{~mm}$ resolution) were acquired for each subject. Functional images were acquired with a reverse spiral imaging sequence $(\mathrm{TR}=2000 \mathrm{msec}, \mathrm{TE}=25 \mathrm{msec}$, flip angle $=$ $75^{\circ}, 32$ slices with $3 \times 3 \times 3$-mm resolution). The first five functional images were discarded from the analysis to allow for steady-state magnetization.

All functional images were slice-time corrected, spatially aligned, and normalized using the normalization parameters used to warp the high-resolution T1 image to the SPM template. After being resampled to a resolution of $2 \times 2 \times 2 \mathrm{~mm}$, they were smoothed with an isotropic 8-mm FWHM Gaussian kernel and high-pass filtered (cutoff $=128 \mathrm{sec})$. For each subject, a statistical model was computed by applying a canonical HRF combined with time and dispersion derivatives for each of the conditions. For the purpose of this control experiment, only the $f /(b+1)$ model was estimated. Analogous to the main experiment, parametric modulators were used that relate the functional data in GTs and STs to the RT pattern in the subsequent GTs (again, only correct GTs and STs that were followed by a correct GT were modeled, whereas all other trial types were modeled separately as regressors of no interest). With respect to the relatively low number of STs in the control experiment and the fact that the degree of RT slowing in GTs after SST and UST was again nearly identical (see Results), the analysis did not differentiate between GTs after SST and UST. The functional data in Figure 3 is displayed on the average of the normalized proton density images of the individual subjects. 


\section{RESULTS}

\section{Behavioral Results}

Subjects performed very accurately on GTs (error rate $=$ 1.4\%) while also being successful in inhibiting their behavioral response on $51 \%$ of the STs. As is typical for this paradigm, responses were faster on USTs than that on GTs ( 455 vs. $493 \mathrm{msec}$ ), $t(11)=19.2, p<.001$.

In addition, the RT data of the present experiment confirmed previous reports of response slowing to GTs following STs versus following GTs ( $484 \mathrm{msec}$ ), $t(11)=2.6, p=.026$. Notably, response slowing on GTs following STs was independent of whether stopping on the preceding trial was successful or not (ssTGT: 499 msec vs. usT GT: 497 msec, $p>$.8).

\section{fMRI Results}

Given that cognitive control is thought to arise as a consequence of error commission or of the detection of response conflict (Kerns et al., 2004) and given the theorized involvement of the SN and/or VTA in cognitive control, we hypothesized that a conflict-driven control signal from these midbrain areas arises in response to STs, which then entails stronger cognitive control, typically associated with slowed responses in the subsequent GT. Importantly, in keeping with theoretical accounts that emphasize the similarity between general cognitive control and reinforcement learning, where reduced dopamine neuron activity is assumed to lead to behavioral adaptation, we hypothesized an inverse relationship between RTs in ${ }_{S T} G T$ and activity in the SN or VTA during the preceding ST.

To test this hypothesis, the hemodynamic response for a given trial was estimated on the basis of its covariation with the behavioral performance of the subsequent GT, separately for GTs, SSTs, and USTs $(f /(b+1)$ model $)$. In addition, a model relating functional activity to RT variations in the same trial (" $\mathrm{f} / \mathrm{b}$ " model) was tested (see below). Importantly, in that we hypothesized that a putative control signal from the midbrain during STs triggers the behavioral adjustments in subsequent GTs, its effect should be visible only when relating the level of functional activity elicited by STs (but not by GTs) to the degree of RT prolongation on the subsequent GT $(f /(b+1)$ model), whereas no such relationship should be present within a trial $(f / b$ model).

We first report the results of the $f /(b+1)$ model. This analysis revealed a significant relationship between the hemodynamic response in STs and the RT in the subsequent $\mathrm{GT}$ in three different brain regions: the right $\mathrm{SN}$ (Figure 2A), the left insula, and the ACC (see Table 1). Importantly, in all three regions, the variation of the hemodynamic response was inversely related to response speed on the subsequent GT, with the largest effect seen in the $\mathrm{SN}$. That is, increased RTs on GTs corresponded with decreased activity during the preceding STs. Notably, the $f /(b+1)$ model did not yield any significant positive

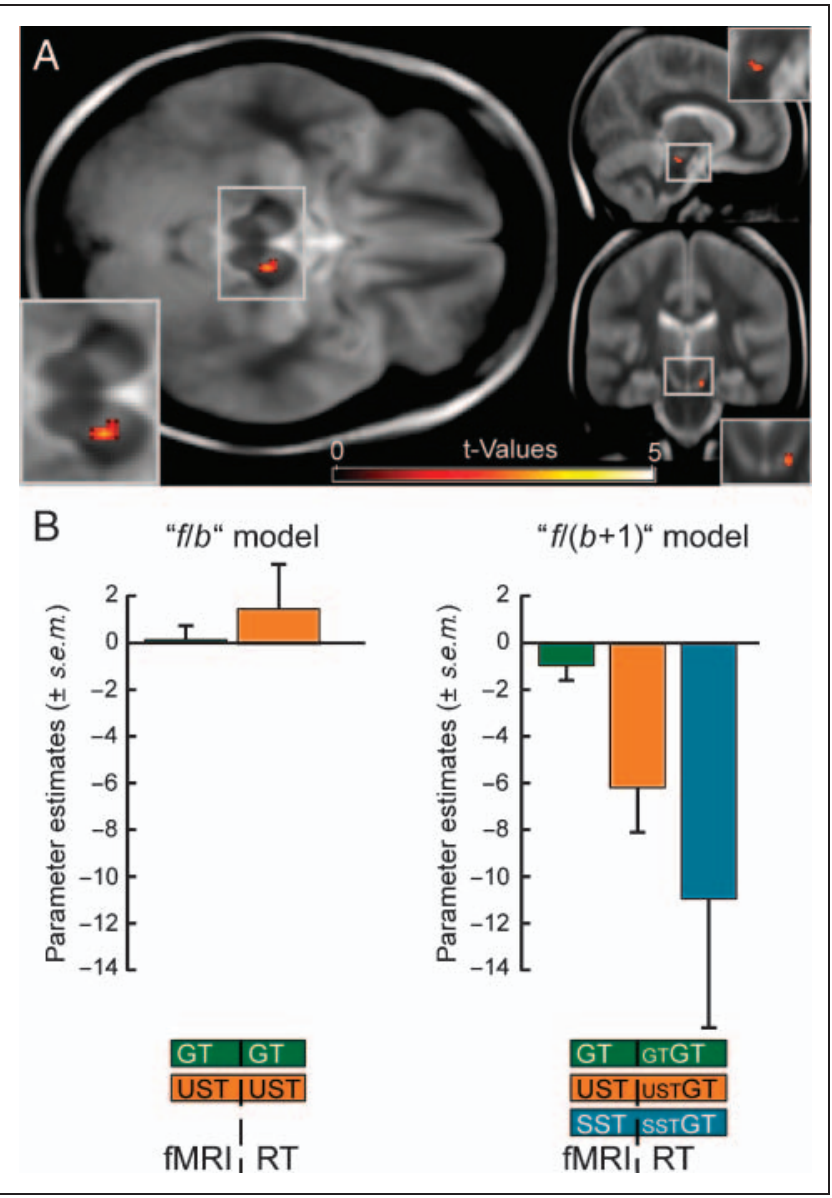

Figure 2. Results of the RT regressor analysis (mean over subjects). (A) The $f /(b+1)$ model revealed a negative relationship between hemodynamic responses in STs and RT fluctuations in the subsequent GTs ( ${ }_{\mathrm{ST}} \mathrm{GT}$ ) within the right SN (MNI coordinates of local activity maximum: $x, y, z=10,-22,-20$ ). (B) ROI analyses revealed that this effect was only present for the RT regressors of the GTs following unsuccessful and SSTs (usT GT and SST GT) in the $f /(b+1)$ model.

correlative relationship in any region of the acquired partial volume with the RT of the next trial.

\section{ROI Analysis}

To provide a more focused overview over the relation between the RT variation and the hemodynamic response in the $\mathrm{SN}$, an ROI analysis was performed (Figure 2B; the ROI was constructed using the average of all three parametric modulators in the $f /(b+1)$ model (GTGT, UST GT, ${ }_{\text {SST }} \mathrm{GT}$ ); see Methods). In the $f /(b+1)$ model, parameter estimates for the RT regressors ${ }_{\text {UST }}$ GT and ${ }_{\text {SST GT but not for }}$ ${ }_{\text {GT }}$ GT significantly differed from zero: ${ }_{\mathrm{UST}} \mathrm{GT}, t(11)=-3.1$, $p=.005 ;$ ssT GT, $t(11)=-2, p=.038 ;{ }_{\mathrm{GT}} \mathrm{GT}, p>.1$. Furthermore, a direct comparison of the RT regressors for

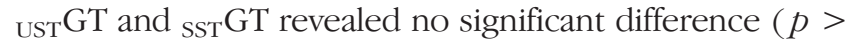
.2), whereas activity estimates both for ${ }_{\mathrm{UST}} \mathrm{GT}$ and ${ }_{\mathrm{SST}} \mathrm{GT}$ were significantly enhanced as compared with ${ }_{\text {GT GT }}$, $t(11)=-2.2, p=.024$ and $t(11)=-1.9, p=.041$. To preview the results of the within-trial analysis in this region, 
Table 1. fMRI Activations during STs Displaying a Negative Relationship to RTs in the Subsequent GT (sTGT; $f /(b+1)$ Model)

\begin{tabular}{|c|c|c|c|c|c|c|}
\hline \multirow{2}{*}{$\begin{array}{l}\text { Anatomical } \\
\text { Structure }\end{array}$} & \multirow[b]{2}{*}{ Hemisphere } & \multirow{2}{*}{$\begin{array}{c}\text { Cluster Size } \\
\text { (Voxel) }\end{array}$} & \multirow[b]{2}{*}{$T$} & \multicolumn{3}{|c|}{ Peak Coordinates MNI ( $\mathrm{mm})$} \\
\hline & & & & $x$ & $y$ & $z$ \\
\hline $\mathrm{SN}^{*}$ & $\mathrm{R}$ & 4 & 7.2 & 10 & -22 & -20 \\
\hline Insula & $\mathrm{L}$ & 10 & 6.64 & -36 & -2 & 4 \\
\hline ACC & $\mathrm{L} / \mathrm{R}$ & 10 & 5.66 & -2 & 18 & 26 \\
\hline
\end{tabular}

Data are thresholded at $p<.001$ (uncorrected), with a cluster level of $k=10$ and $* k=4$ in the midbrain (SVC-corrected $p=.018$ for the SN cluster). Neither of the cortical activations were significant on the cluster level $(p<.05)$ after correction for multiple comparisons with respect to the whole acquired partial volume.

the $f / b$ model did not yield any significant relationship between activity in the SN and the RT in a given trial (see below). Consistently, the $f / b$ model did not yield significant estimates related to RT regressors for GT and UST in the SN ROI (both $p>.4$ ), suggesting that activity in the SN on a given trial has negligible influence on RT performance on that trial.

\section{Control Experiment}

It has been argued that the midbrain is particularly difficult to image with fMRI (D'Ardenne, McClure, Nystrom, \& Cohen, 2008; but see Duzel et al., 2009). In fact, our main finding reports only a very small cluster of significant voxels showing the predicted activity pattern. Hence, to extend the data basis of our interpretation, we analyzed data of a similar follow-up experiment that included analogous conditions and permitted to analyze the effects of STs on subsequent GT performance as in the just reported main experiment.

Behaviorally, the RT slowing following STs in the control experiment was even more pronounced than in the main experiment ( ${ }_{\mathrm{GT}} \mathrm{GT}=523 \mathrm{msec}$; $\left.\mathrm{sT}_{\mathrm{GT}}=571 \mathrm{msec}\right), t(15)=$ $5, p<.001$. This slowing, again, did not depend on the success of the previous-trial response inhibition (UsTGT $=$ 570 msec; ${ }_{\text {ssT }} \mathrm{GT}=572 \mathrm{msec} ; p>$.8). Critically, as in the main experiment, an inverse relationship was observed in the SN between the hemodynamic response to STs and the response speed on the subsequent GT (see Figure 3; Montreal Neurological Institute [MNI] coordinates of local activity maximum: $x, y, z=12,-24,-14$; peak $t$ value $=$ 4.76 ; cluster size $=9$ voxels; SVC-corrected $p$ value $=$ .022). This relation was not observed anywhere else (at the threshold level used in the main experiment), and again, no such relationship was observed for GTs following GTs. Thus, the control experiment clearly replicated our main finding that activity in the SN in STs is inversely related to the degree of RT slowing in the subsequent GT.

\section{Fluctuations in GTs}

Given that fluctuations in SN activity on an ST systematically relate to control adjustments on the following trial, but not to performance changes within a trial, one may ask which neural structures are actually related to RT performance within a given trial. To address this, we explored the relationship of brain activity and RTs on a given GT ( $f / b$ model) of the main experiment. A positive relationship, that is, a larger hemodynamic response for longer RTs, was present in a number of cortical areas including lateral frontal, inferior parietal, and precentral regions, the latter coinciding with the primary, supplementary, and premotor
Figure 3. Relationship between neural activity in STs and the RT in the subsequent GT in the control experiment ( $\mathrm{sT}_{\mathrm{GT}} \mathrm{in}$ the $f /(b+1)$ model; mean over subjects). Similar to the main experiment, there was a negative relationship between hemodynamic responses in STs and RT fluctuations in the subsequent GTs within the right SN (MNI coordinates of local activity maximum: $x, y, z=12$, $-24,-14)$.

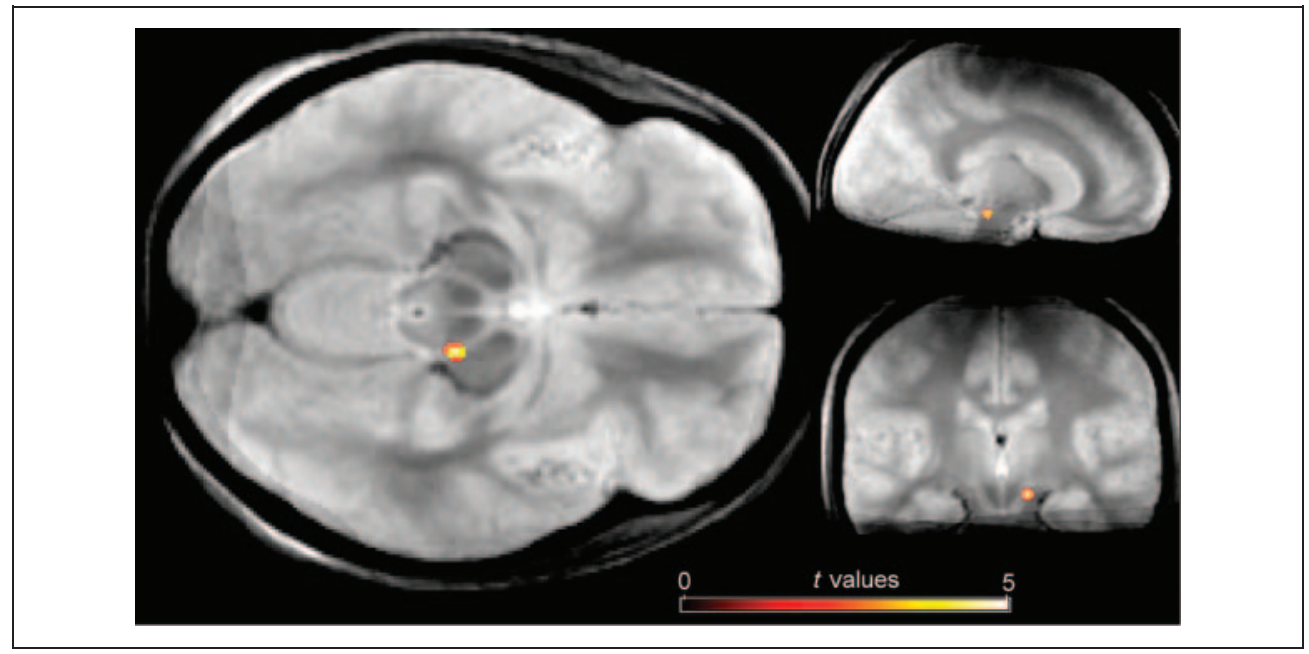


areas (see Table 2). The same relationship was found in the left fusiform gyrus as well as bilaterally in medial frontal areas (pre-SMA) plus the dorsal portion of the ACC (dACC) (Figure $4 \mathrm{~A}$ ) and the insula. In the midbrain, a positive re- lation to RT appeared in a region directly below the right thalamus, likely representing the subthalamic nucleus (STN; Figure 4B; see Aron \& Poldrack, 2006). By contrast, very few regions displayed a negative relationship to RT

Table 2. fMRI Activations Displaying a Positive Relationship to RTs in GTs ( $f / b$ Model)

\begin{tabular}{|c|c|c|c|c|c|c|}
\hline \multirow{2}{*}{$\begin{array}{l}\text { Anatomical } \\
\text { Structure }\end{array}$} & \multirow[b]{2}{*}{ Hemisphere } & \multirow{2}{*}{$\begin{array}{c}\text { Cluster Size } \\
\text { (Voxel) }\end{array}$} & \multirow[b]{2}{*}{$T$} & \multicolumn{3}{|c|}{ Peak Coordinates MNI ( $\mathrm{mm})$} \\
\hline & & & & $x$ & $y$ & $z$ \\
\hline STN* & $\mathrm{R}$ & 7 & 5.32 & 10 & -16 & -2 \\
\hline \multirow[t]{3}{*}{ Inferior parietal cortex** } & $\mathrm{L}$ & 201 & 9.96 & -44 & -32 & 46 \\
\hline & & & 5.5 & -62 & -22 & 34 \\
\hline & & & 5.41 & -66 & -32 & 34 \\
\hline \multirow[t]{3}{*}{ Fusiform gyrus** } & $\mathrm{L}$ & 86 & 9.87 & -36 & -44 & -18 \\
\hline & & & 6.04 & -46 & -44 & -10 \\
\hline & & & 4.94 & -44 & -54 & -8 \\
\hline \multirow[t]{3}{*}{ Precentral gyrus** } & $\mathrm{L}$ & 151 & 7.5 & -26 & -20 & 70 \\
\hline & & & 5.94 & -20 & -12 & 72 \\
\hline & & & 5.9 & -36 & -18 & 62 \\
\hline Insula** & $\mathrm{R}$ & 39 & 7.48 & 38 & 0 & 16 \\
\hline \multirow[t]{3}{*}{ Inferior parietal cortex** } & $\mathrm{R}$ & 111 & 7.25 & 52 & -28 & 40 \\
\hline & & & 6.57 & 64 & -18 & 40 \\
\hline & & & 5.99 & 62 & -24 & 50 \\
\hline Fusiform gyrus & $\mathrm{L}$ & 11 & 7.19 & -30 & -8 & -34 \\
\hline Precentral gyrus & $\mathrm{R}$ & 23 & 7.15 & 40 & -12 & 62 \\
\hline Inferior frontal cortex & $\mathrm{R}$ & 16 & 6.97 & 62 & 14 & 18 \\
\hline \multirow[t]{2}{*}{ Precentral gyrus** } & $\mathrm{L}$ & 90 & 6.77 & -36 & -6 & 46 \\
\hline & & & 5.91 & -48 & 0 & 36 \\
\hline \multirow[t]{3}{*}{ Precentral gyrus** } & $\mathrm{L}$ & 72 & 6.45 & -24 & -8 & 56 \\
\hline & & & 5.26 & -32 & -10 & 54 \\
\hline & & & 4.60 & -26 & -4 & 48 \\
\hline Precentral gyrus & $\mathrm{L}$ & 16 & 5.99 & -50 & -6 & 44 \\
\hline Inferior parietal cortex & $\mathrm{R}$ & 11 & 5.92 & 50 & -28 & 50 \\
\hline \multirow[t]{2}{*}{ Insula** } & $\mathrm{L}$ & 46 & 5.8 & -34 & 16 & 10 \\
\hline & & & 4.44 & -30 & 10 & 16 \\
\hline \multirow[t]{2}{*}{ Superior frontal gyrus** } & $\mathrm{R}$ & 31 & 5.58 & 28 & -8 & 54 \\
\hline & & & 5.43 & 26 & -4 & 64 \\
\hline Pre-SMA/cingulate cortex** & $\mathrm{L} / \mathrm{R}$ & 69 & 5.57 & 2 & 14 & 50 \\
\hline Inferior frontal gyrus & $\mathrm{R}$ & 11 & 4.9 & 36 & 6 & 30 \\
\hline Insula & $\mathrm{L}$ & 14 & 4.75 & -38 & 4 & 6 \\
\hline Inferior frontal gyrus** & $\mathrm{L}$ & 27 & 4.64 & -42 & 12 & 10 \\
\hline
\end{tabular}

Data are thresholded at $p<.001$ (uncorrected), with a cluster level of $k=10$ and $* k=4$ in the midbrain.

** $p<.05$ on the cluster level after correction for multiple comparisons with respect to the whole acquired partial volume. 
Figure 4. Areas showing a significant within-trial correlation for GTs ( $f / b$ model; mean over subjects). A positive relationship between RTs and hemodynamic response in GTs, that is, stronger activity for dACC/pre-SMA (A; MNI coordinates of local activity maximum: $x, y, z=2,14,50$ ), along with several other areas and the STN (B; MNI coordinates of local activity longer RTs, was present in the maximum: $x, y, z=10,-16,-2)$.

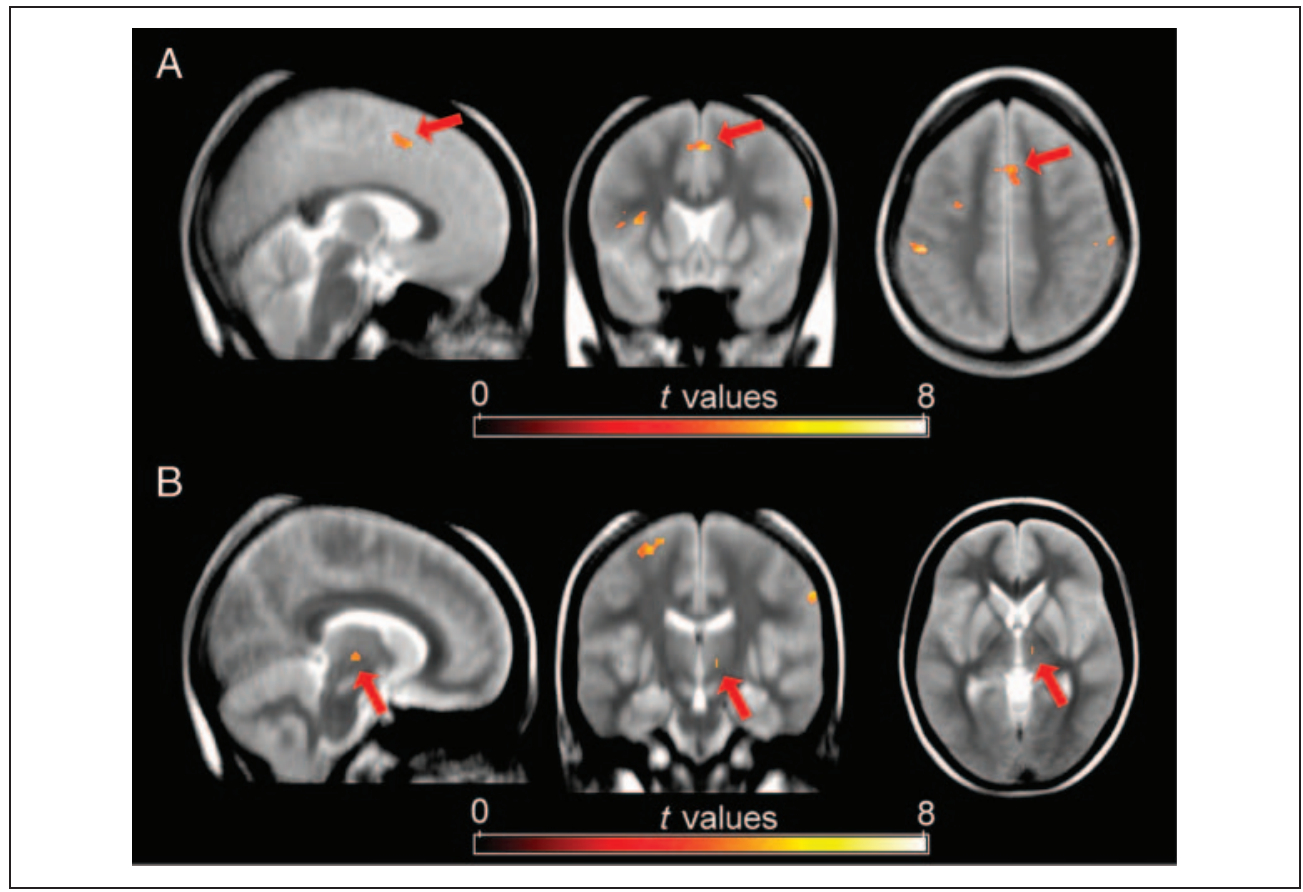

within trial (see Table 3), which were essentially confined to the $\mathrm{BG}$ and the thalamus.

\section{DISCUSSION}

The fMRI data reported here indicate that under conditions that tax cognitive control, activity changes in the SN link in a systematic way to response speed on the subsequent trial (lower SN activity in an ST being associated with longer RTs and thus presumably greater cognitive control in a subsequent GT). This notable pattern of results was replicated in a second experiment. Hence, activity in the $\mathrm{SN}$ in response to an $\mathrm{ST}$ is predictive of subsequent behavioral adjustments. It is important to emphasize that this predictive link was only observed for GTs following STs and not for GTs following GTs. This indicates that the inverse relationship between SN activity and future performance arises as a consequence of STs, presumably attributable to the inherent response conflict elicited by the opposing tendencies of initiating versus withholding a response. We assume, however, that a similar pattern of results would also be obtained for simple performance errors like incorrect GTs. Unfortunately, these could not be investigated because of their small number. Notably,

Table 3. fMRI Activations Displaying a Negative Relationship to RTs in GTs ( $f / b$ Model)

\begin{tabular}{|c|c|c|c|c|c|c|}
\hline \multirow{2}{*}{$\begin{array}{l}\text { Anatomical } \\
\text { Structure }\end{array}$} & \multirow[b]{2}{*}{ Hemisphere } & \multirow{2}{*}{$\begin{array}{c}\text { Cluster Size } \\
\text { (Voxel) }\end{array}$} & \multirow[b]{2}{*}{$T$} & \multicolumn{3}{|c|}{ Peak Coordinates MNI ( $\mathrm{mm})$} \\
\hline & & & & $x$ & $y$ & $z$ \\
\hline \multirow[t]{3}{*}{ Pallidum/putamen* } & $\mathrm{R}$ & 45 & 8.79 & 20 & 4 & -8 \\
\hline & & & 5.51 & 22 & 12 & -4 \\
\hline & & & 4.21 & 26 & 2 & -2 \\
\hline Thalamus* & $\mathrm{L}$ & 89 & 7.17 & -2 & -8 & 12 \\
\hline \multirow[t]{3}{*}{ Pallidum* } & $\mathrm{L}$ & 69 & 6.49 & -22 & -6 & -6 \\
\hline & & & 6.47 & -14 & 8 & -4 \\
\hline & & & 6.23 & -18 & 0 & -2 \\
\hline Mid-frontal gyrus & $\mathrm{L}$ & 10 & 5.56 & -38 & 18 & 52 \\
\hline Cerebellum & $\mathrm{L}$ & 21 & 5.25 & -2 & -48 & -36 \\
\hline Hippocampus & $\mathrm{L}$ & 15 & 5.05 & -36 & -18 & -8 \\
\hline
\end{tabular}

Data are thresholded at $p<.001$ (uncorrected), with a cluster level of $k=10$ (no midbrain activity at $k=4$ ).

${ }^{*} p<.05$ on the cluster level after correction for multiple comparisons with respect to the whole acquired partial volume. 
activity fluctuations in the SN did not correlate with the subjects' performance within the same trial. This pattern of results is highly compatible with the notion that the SN provides a varying control signal upon response conflict to adjust subsequent cognitive control.

Our results clearly speak in favor of suggestions that activity in the midbrain modulates cortical and subcortical regions that mediate cognitive control, potentially sharing this mechanism with reward-dependent reinforcement learning (Brown \& Braver, 2005; Frank et al., 2005; Montague et al., 2004; Ridderinkhof, Ullsperger, et al., 2004; Ridderinkhof, van den Wildenberg, et al., 2004; Holroyd \& Coles, 2002). In reinforcement learning, dopaminergic neurons in the midbrain are thought to convey a "teaching signal" to the BG and the frontal cortex, with reward being coded as an increase and its omission as a decrease in dopaminergic transmission. It is assumed that the former leads to a perseverance of rewarded actions whereas the latter causes a change in behavior (Schultz, 2000). Several computational models have proposed that a very similar mechanism might underlie cognitive control in the absence of reward, with conditions that lead to stronger subsequent cognitive control in general, and the commission of errors in particular, being taken as equivalent to the omission of reward (Brown \& Braver, 2005; Frank et al., 2005; Holroyd \& Coles, 2002; Braver $\&$ Cohen, 2000). Thus, following those lines of interpretation, conditions of increased cognitive control can be expected to be preceded by reduced activity in the SN or VTA. In the present studies, this appears to be reflected in the linear negative relationship between the SN activity during an ST and the subsequent GT RT prolongation.

The present observations may also be discussed in relation to recent pharmacological observations in rodents. Potentially paralleling the present data, Bari, Eagle, Mar, Robinson, and Robbins (2009) observed that lower levels of dopamine were accompanied by slower responses in the rodent. On the other hand, dopamine does not appear to play a role in the actual stopping process, as stopping seems to be rather influenced by noradrenaline, suggestive of a functional dissociation of neuromodulation related to the go and stop process (Eagle, Tufft, Goodchild, \& Robbins, 2007).

Our observations also match well with reports of differential post-non-inhibition/post-error slowing because of genetic polymorphisms in the dopaminergic system (Kramer et al., 2007) or psychopharmacological interventions thereof (Zirnheld et al., 2004), which were both accompanied by concomitant variations of the errorrelated-negativity ERP component (see also Klein et al., 2007). However, error processing per se did not appear to be the crucial feature underlying the $\mathrm{SN}$ activity in our study, as both SSTs and UST displayed a similar relationship between SN activity changes and subsequent RT prolongation. One possibility to reconcile the current data with those previous findings may be the idea that the SN provides a control signal related to general response con- flict or error likelihood, for which actual errors represent only a special case (Brown \& Braver, 2005; Yeung, Cohen, \& Botvinick, 2004; Botvinick et al., 2001). In support of the idea that dopaminergic structures play such a role in conflict-driven behavioral adaptation, it has been demonstrated that patients suffering from Parkinson's disease display markedly reduced or no behavioral adaptation after high-conflict trials in the Simon task (Fielding, GeorgiouKaristianis, Bradshaw, Millist, \& White, 2005; Praamstra \& Plat, 2001). Importantly, this effect was independent of actual task errors. Moreover, a recent behavioral study that investigated the influence of reward on conflict adaptation (van Steenbergen, Band, \& Hommel, 2009) reported that conflict-related RT adjustments from one trial to the next were abolished when subjects received a monetary reward in-between. This finding is consistent with the notion that a dopaminergic response to the reward could overrule the dopaminergic modulations that may have been engaged to adapt behavior between trials in response to response conflict.

With respect to the accounts of response conflict, one might argue that the stop-signal paradigm does not represent one of the typical conflict paradigms (like the Stroop, Flanker, or Simon task), thus putting it at some distance from the above explanation. We note, however, that the stop-signal paradigm involves a high degree of response conflict because the tendencies of going and stopping are at direct odds with each other. In addition, on-line performance tracking constantly keeps this task in a very challenging range. Importantly, our observations argue against explanations of the observed RT prolongation in terms of a general "inhibitory aftereffect" (Rieger \& Gauggel, 1999). One could argue that inhibitory processes of the motor system in STs, by virtue of an inherent slowness, spill into the successive trial, thereby slowing performance. Although this interpretation might fit with the behavioral observation, it does not explain the pattern of brain activity we found. In particular, there is no indication of the SN being involved in response speed or response inhibition per se, as indicated by the lack of a significant relationship to RTs within trials. Furthermore, such inhibitory aftereffects would be expected throughout the motor system and not only in the $\mathrm{SN}$ - a pattern not observed here.

Another important issue pertains to the broader systems-level context, in which the SN signal arises and exerts its influence and consequently to the precise role that the SN plays in the larger process. It has been demonstrated that ACC plays an important role during the detection of conflict, which appears to be important for subsequent adaptation (e.g., Kerns et al., 2004). The present study, however, found a more robust relationship to the subsequent behavior in the SN. Nonetheless, this should not be taken to indicate that ACC does not play an important role in the process but rather that it might do so in a fashion that does not result in an equally strong linear relationship between its activity level and the subsequent behavioral adjustment. Although the exact functional 
relationship between ACC and the $\mathrm{SN}$ is not yet clear, the existence of bidirectional connections between these two areas (Seamans \& Yang, 2004; Carr \& Sesack, 2000), along with influential models proposing a tight functional link between them (e.g., Holroyd \& Coles, 2002), suggests that they act in some sort of joint manner. The precise nature of this interaction and thus their respective roles and activation sequence under varying conditions remain to be determined. In our view, it seems likely that the central function subserved by the $\mathrm{SN}$ is to link the detection of a need for a behavioral adjustment in a given trial to the actual implementation of that adjustment in the subsequent trial, providing a bridge across time in the process.

In the present study, when examining the determinants of response speed in GTs (i.e., the $f / b$ model), a widespread network of cortical and subcortical structures was identified. In the stop-signal paradigm, GTs always have the potential to turn into STs, thus necessitating titration of the optimal RT (Verbruggen \& Logan, 2009; Jaffard et al., 2008; Vink et al., 2005). Thus, it does not seem surprising that most areas that were identified displayed a positive within-trial relationship to RT (stronger activity for longer RTs). Other studies, however, also reported positive correlations between RT and various brain areas that might not necessarily be related to an active delaying mechanism (Yarkoni et al., 2009; Weissman et al., 2006). In the current study, areas in the frontal, insular, and parietal cortex were more active for long RTs in the within-GT analysis, as were various motor areas, the fusiform gyrus, the dACC/pre-SMA, and the STN. The opposite relationship was found in parts of the BG and the thalamus. It is not possible in the current study to pinpoint the actual locus where the control signal from the midbrain (elicited by a preceding ST) impacts this network. However, there are known projections from the SN to the medial frontal cortex, including the dACC/pre-SMA, that area thought to serve modulatory functions (Quilodran, Rothe, \& Procyk, 2008). In fact, dACC/pre-SMA has previously been implicated in post-error slowing (Marco-Pallares, Camara, Munte, \& Rodriguez-Fornells, 2008; Debener et al., 2005), which may be accomplished by influencing the lateral frontal cortex to actually change neural processing in the subsequent trial (Li et al., 2008; Kerns et al., 2004). Alternatively or in addition, SN activity might influence the activity in the striatum and the STN, as both receive inputs from the SN and have been implicated in response inhibition (Frank, Samanta, Moustafa, \& Sherman, 2007; Kempf et al., 2007; Frank, 2006; Vink et al., 2005). A role of the STN has been explicitly demonstrated for inhibitory motor control in a stop-signal paradigm (Aron, Behrens, Smith, Frank, \& Poldrack, 2007; Aron \& Poldrack, 2006). Potentially, the STN may not only be engaged for outright stopping of a motor response, as suggested by these studies, but might also be engaged to exert a global no-go-signal on the BG that "buys time" to further elaborate on a response in the sense of a time-accuracy trade-off (Frank et al., 2007; Frank, 2006). Concerning activity in the STN in the present study, some caution has to be applied because we did not have a specific a priori hypothesis, and its activity did not survive family-wise error correction. We think, however, in view of the theoretical framework presented above, it is not unlikely that the STN was indeed active in the reported contrast.

Clearly, SN activity cannot be equated to dopaminergic transmission in the target areas (Seamans \& Yang, 2004), and animal physiology has started to discover that different dopamine neurons react differently to positive and negative reinforcers (Matsumoto \& Hikosaka, 2009). On the relatively coarse level of human fMRI studies, however, studies investigating reward have demonstrated effects bearing the signature of well-described reward-related dopaminergic mechanisms seen in animals (e.g., D'Ardenne et al., 2008; Wittmann et al., 2005). Furthermore, a recent study using PET/fMRI in parallel also speaks in favor of a strong relationship between SN/VTA activity and dopaminergic neurotransmission (Schott et al., 2008). An additional, relatively indirect indication derives from the delayed timing with which the activity in the SN seems to impact behavioral performance in this study, which appears to be consistent with a slower neuromodulatory mechanism (Seamans \& Yang, 2004). It therefore appears likely that the effects demonstrated in this study reflect at least in part the dopaminergic output of the SN.

Finally, our fMRI data do not allow to unequivocally distinguish between the SN pars compacta (that contains the majority of dopaminergic neurons in humans) and the SN pars reticulata. On the one hand, this is due to the limited spatial resolution, but also because the two structures are highly interwoven, especially in humans (for a discussion of using fMRI to investigate the dopaminergic midbrain structures in humans, see Duzel et al., 2009). The SN pars reticulata, however, has also been implicated in cognitive functions that might bear to some extent on the interpretation of the present data (Frank, Loughry, \& O'Reilly, 2001). With respect to the theoretical framework provided by different models of the involvement of dopamine in cognitive control, however, we believe that the $\mathrm{SN}$ pars compacta is the more likely neural substrate in the present study.

Taken together, our data indicate that under high demands for maintaining flexible cognitive control, activity in the SN becomes predictive of future performance, with decreased activity leading to longer RTs in the subsequent trial. We suggest that this conditional dependency refers to the operation of a dopaminergic control signal that bears strong similarities to the dopaminergic "teaching signal" previously reported in reward-dependent reinforcement learning. A disturbance of this signal might diminish the ability to flexibly adapt one's behavior, which is a psychopathological feature of a number of neuropsychiatric disorders (Montague et al., 2004; Nieoullon, 2002). Our findings might therefore provide new insights into the mechanistic dysfunctions underlying these conditions. 


\section{Acknowledgments}

This research was funded by German grants from the BMBF (contract no. 01GO0202) to the Center for Advanced Imaging, Magdeburg, the DFG to T. N. (SFB-TR31/TPA8), to C. N. B. (BO 3345/1-1), and to T. F. M., M. A. S., J. M. H., and H. J. H. (SFB 779), and by a U.S. grant from the NIH (R01-NS051048) to M. G. W.

Reprint requests should be sent to Dr. Carsten Nicolas Boehler, Center for Cognitive Neuroscience, Box 90999, Duke University, LSRC Bldg., Rm. B203, Durham, NC 27708-0999, or via e-mail: nico.boehler@duke.edu.

\section{REFERENCES}

Aron, A. R., Behrens, T. E., Smith, S., Frank, M. J., \& Poldrack, R. A. (2007). Triangulating a cognitive control network using diffusion-weighted magnetic resonance imaging (MRI) and functional MRI. Journal of Neuroscience, 27, 3743-3752.

Aron, A. R., \& Poldrack, R. A. (2006). Cortical and subcortical contributions to stop signal response inhibition: Role of the subthalamic nucleus. Journal of Neuroscience, 26, 2424-2433.

Ashburner, J., \& Friston, K. J. (1999). Nonlinear spatial normalization using basis functions. Human Brain Mapping, 7, 254-266.

Bari, A., Eagle, D. M., Mar, A. C., Robinson, E. S., \& Robbins, T. W. (2009). Dissociable effects of noradrenaline, dopamine, and serotonin uptake blockade on stop task performance in rats. Psychopharmacology (Berlin), 205, 273-283.

Boehler, C. N., Munte, T. F., Krebs, R. M., Heinze, H. J., Schoenfeld, M. A., \& Hopf, J. M. (2009). Sensory MEG responses predict successful and failed inhibition in a stop-signal task. Cerebral Cortex, 19, 134-145.

Botvinick, M. M., Braver, T. S., Barch, D. M., Carter, C. S., \& Cohen, J. D. (2001). Conflict monitoring and cognitive control. Psychological Review, 108, 624-652.

Botvinick, M. M., Cohen, J. D., \& Carter, C. S. (2004). Conflict monitoring and anterior cingulate cortex: An update. Trends in Cognitive Sciences, 8, 539-546.

Braver, T. S., \& Cohen, J. D. (2000). On the control of control: The role of dopamine in regulating prefrontal function and working memory. In S. Monsell \& J. Driver (Eds.), Attention and performance XVIII (pp. 713-738). Cambridge, MA: MIT Press.

Brown, J. W., \& Braver, T. S. (2005). Learned predictions of error likelihood in the anterior cingulate cortex. Science, 307, 1118-1121.

Buchel, C., Holmes, A. P., Rees, G., \& Friston, K. J. (1998). Characterizing stimulus-response functions using nonlinear regressors in parametric fMRI experiments. Neuroimage, 8, 140-148.

Bunzeck, N., \& Duzel, E. (2006). Absolute coding of stimulus novelty in the human substantia nigra/VTA. Neuron, 51, 369-379.

Carr, D. B., \& Sesack, S. R. (2000). Projections from the rat prefrontal cortex to the ventral tegmental area: Target specificity in the synaptic associations with mesoaccumbens and mesocortical neurons. Journal of Neuroscience, 20, 3864-3873.

Carter, C. S., Braver, T. S., Barch, D. M., Botvinick, M. M., Noll, D., \& Cohen, J. D. (1998). Anterior cingulate cortex, error detection, and the online monitoring of performance. Science, 280, 747-749.

D’Ardenne, K., McClure, S. M., Nystrom, L. E., \& Cohen, J. D. (2008). BOLD responses reflecting dopaminergic signals in the human ventral tegmental area. Science, 319, 1264-1267.

Debener, S., Ullsperger, M., Siegel, M., Fiehler, K., von Cramon, D. Y., \& Engel, A. K. (2005). Trial-by-trial coupling of concurrent electroencephalogram and functional magnetic resonance imaging identifies the dynamics of performance monitoring. Journal of Neuroscience, 25, 11730-11737.

Duzel, E., Bunzeck, N., Guitart-Masip, M., Wittmann, B., Schott, B. H., \& Tobler, P. N. (2009). Functional imaging of the human dopaminergic midbrain. Trends in Neurosciences, 32, 321-328.

Eagle, D. M., Tufft, M. R., Goodchild, H. L., \& Robbins, T. W. (2007). Differential effects of modafinil and methylphenidate on stop-signal reaction time task performance in the rat, and interactions with the dopamine receptor antagonist cis-flupenthixol. Psychopharmacology (Berlin), 192, 193-206.

Enticott, P. G., Bradshaw, J. L., Bellgrove, M. A., Upton, D. J., \& Ogloff, J. R. (2009). Stop task after-effects. Experimental Psychology, 56, 247-251.

Fielding, J., Georgiou-Karistianis, N., Bradshaw, J., Millist, L., \& White, O. (2005). No sequence dependent modulation of the Simon effect in Parkinson's disease. Brain Research, Cognitive Brain Research, 25, 251-260.

Frank, M. J. (2006). Hold your horses: A dynamic computational role for the subthalamic nucleus in decision making. Neural Networks, 19, 1120-1136.

Frank, M. J., Loughry, B., \& O’Reilly, R. C. (2001). Interactions between frontal cortex and basal ganglia in working memory: A computational model. Cognitive, Affective \& Behavioral Neuroscience, 1, 137-160.

Frank, M. J., Samanta, J., Moustafa, A. A., \& Sherman, S. J. (2007). Hold your horses: Impulsivity, deep brain stimulation, and medication in parkinsonism. Science, 318, 1309-1312.

Frank, M. J., Woroch, B. S., \& Curran, T. (2005). Error-related negativity predicts reinforcement learning and conflict biases. Neuron, 47, 495-501.

Friston, K. J., Fletcher, P., Josephs, O., Holmes, A., Rugg, M. D., \& Turner, R. (1998). Event-related fMRI: Characterizing differential responses. Neuroimage, 7, 30-40.

Gehring, W. J., \& Knight, R. T. (2000). Prefrontal-cingulate interactions in action monitoring. Nature Neuroscience, 3, 516-520.

Holroyd, C. B., \& Coles, M. G. (2002). The neural basis of human error processing: Reinforcement learning, dopamine, and the error-related negativity. Psychological Review, 109, 679-709.

Jaffard, M., Longcamp, M., Velay, J. L., Anton, J. L., Roth, M., Nazarian, B., et al. (2008). Proactive inhibitory control of movement assessed by event-related fMRI. Neuroimage, 42, 1196-1206.

Kempf, F., Brucke, C., Kuhn, A. A., Schneider, G. H., Kupsch, A., Chen, C. C., et al. (2007). Modulation by dopamine of human basal ganglia involvement in feedback control of movement. Current Biology, 17, R587-R589.

Kerns, J. G., Cohen, J. D., MacDonald, A. W., III, Cho, R. Y., Stenger, V. A., \& Carter, C. S. (2004). Anterior cingulate conflict monitoring and adjustments in control. Science, 303, 1023-1026.

Klein, T. A., Neumann, J., Reuter, M., Hennig, J., von Cramon, D. Y., \& Ullsperger, M. (2007). Genetically determined differences in learning from errors. Science, 318, 1642-1645.

Kramer, U. M., Cunillera, T., Camara, E., Marco-Pallares, J., Cucurell, D., Nager, W., et al. (2007). The impact of catechol-O-methyltransferase and dopamine D4 receptor genotypes on neurophysiological markers of performance monitoring. Journal of Neuroscience, 27, 14190-14198. 
Li, C. S., Huang, C., Yan, P., Paliwal, P., Constable, R. T., \& Sinha, R. (2008). Neural correlates of posterror slowing during a stop signal task: A functional magnetic resonance imaging study. Journal of Cognitive Neuroscience, 20, 1021-1029.

Logan, G. D. (1994). On the ability to inhibit thought and action: A user's guide to the stop signal paradigm. In D. Dagenbach \& T. H. Carr (Eds.), Inbibitory processes in attention, memory, and language (pp. 189-239). San Diego: Academic Press.

Marco-Pallares, J., Camara, E., Munte, T. F., \& RodriguezFornells, A. (2008). Neural mechanisms underlying adaptive actions after slips. Journal of Cognitive Neuroscience, 20, $1595-1610$.

Matsumoto, M., \& Hikosaka, O. (2009). Two types of dopamine neuron distinctly convey positive and negative motivational signals. Nature, 459, 837-841.

Montague, P. R., Hyman, S. E., \& Cohen, J. D. (2004). Computational roles for dopamine in behavioural control. Nature, 431, 760-767.

Nieoullon, A. (2002). Dopamine and the regulation of cognition and attention. Progress in Neurobiology, 67, 53-83.

Pasupathy, A., \& Miller, E. K. (2005). Different time courses of learning-related activity in the prefrontal cortex and striatum. Nature, 433, 873-876.

Praamstra, P., \& Plat, F. M. (2001). Failed suppression of direct visuomotor activation in Parkinson's disease. Journal of Cognitive Neuroscience, 13, 31-43.

Quilodran, R., Rothe, M., \& Procyk, E. (2008). Behavioral shifts and action valuation in the anterior cingulate cortex. Neuron, 57, 314-325.

Ridderinkhof, K. R., Ullsperger, M., Crone, E. A., \& Nieuwenhuis, S. (2004). The role of the medial frontal cortex in cognitive control. Science, 306, 443-447.

Ridderinkhof, K. R., van den Wildenberg, W. P. M., Segalowitz, S. J., \& Carter, C. S. (2004). Neurocognitive mechanisms of cognitive control: The role of prefrontal cortex in action selection, response inhibition, performance monitoring, and reward-based learning. Brain and Cognition, 56, 129-140.

Rieger, M., \& Gauggel, S. (1999). Inhibitory after-effects in the stop signal paradigm. British Journal of Psychology, 90, 509-518.

Schott, B. H., Minuzzi, L., Krebs, R. M., Elmenhorst, D., Lang, M., Winz, O. H., et al. (2008). Mesolimbic functional magnetic resonance imaging activations during reward anticipation correlate with reward-related ventral striatal dopamine release. Journal of Neuroscience, 28, 14311-14319.

Schultz, W. (2000). Multiple reward signals in the brain. Nature Reviews Neuroscience, 1, 199-207.

Seamans, J. K., \& Yang, C. R. (2004). The principal features and mechanisms of dopamine modulation in the prefrontal cortex. Progress in Neurobiology, 74, 1-58.

van Steenbergen, H., Band, G. P., \& Hommel, B. (2009). Reward counteracts conflict adaptation: Evidence for a role of affect in executive control. Psychological Science, 20, 1473-1477.

Verbruggen, F., \& Logan, G. D. (2009). Models of response inhibition in the stop-signal and stop-change paradigms. Neuroscience and Biobehavioral Reviews, 33, 647-661.

Vink, M., Kahn, R. S., Raemaekers, M., van den Heuvel, M., Boersma, M., \& Ramsey, N. F. (2005). Function of striatum beyond inhibition and execution of motor responses. Human Brain Mapping, 25, 336-344.

Weissman, D. H., Roberts, K. C., Visscher, K. M., \& Woldorff, M. G. (2006). The neural bases of momentary lapses in attention. Nature Neuroscience, 9, 971-978.

Wittmann, B. C., Schott, B. H., Guderian, S., Frey, J. U., Heinze, H. J., \& Duzel, E. (2005). Reward-related fMRI activation of dopaminergic midbrain is associated with enhanced hippocampus-dependent long-term memory formation. Neuron, 45, 459-467.

Worsley, K. J., Marrett, S., Neelin, P., Vandal, A. C., Friston, K. J., \& Evans, A. C. (1996). A unified statistical approach for determining significant signals in images of cerebral activation. Human Brain Mapping, 4, 58-73.

Yarkoni, T., Barch, D. M., Gray, J. R., Conturo, T. E., \& Braver, T. S. (2009). BOLD correlates of trial-by-trial reaction time variability in gray and white matter: A multi-study fMRI analysis. PLOS ONE, 4, e4257.

Yeung, N., Cohen, J. D., \& Botvinick, M. M. (2004). The neural basis of error detection: Conflict monitoring and the error-related negativity. Psychological Review, 111, 931-959.

Zirnheld, P. J., Carroll, C. A., Kieffaber, P. D., O’Donnell, B. F., Shekhar, A., \& Hetrick, W. P. (2004). Haloperidol impairs learning and error-related negativity in humans. Journal of Cognitive Neuroscience, 16, 1098-1112. 